Reprod. Nutr. Develop., 1988, 28 (3 B), 773-780

\title{
Innervation, rythme d'activité et accumulation des chaînes légères de myosine d'un muscle rapide de poulet
}

\author{
Marie-France GARDAHAUT, A. KHASKIYE, T. ROUAUD, D. RENAUD, \\ G. LE DOUARIN
}

Groupe de Physiologie Cellulaire, Centre de Recherche de Biologie et Physico-Chimie Cellulaires, Faculté des Sciences, 2, rue de la Houssinière, 44072 Nantes Cedex 03. France.

Summary. Innervation, activity rhythm and accumulation of myosin light chains of fast muscle in chickens.

The fast posterior latissimus dorsi muscle (PLD) of the chick ceases to accumulate slow myosin light chains (MLC) during neonatal development. On day 18 of embryonic life slow MLC represented only $2 \%$ of total $M L C$, and $L^{2} C_{3} F$ was first detected. In chick embryo, spinal cord stimulation at a slow rhythm modifies PLD differentiation toward the slow type : $\mathrm{LC}_{3} \mathrm{~F}$ did not accumulate and slow MLC increased. In constrast, stimulation at a fast rhythm accelerated $L_{3} F$ accumulation. PLD denervation on day 2 after hatching inhibited the synthesis of $\mathrm{LC}_{3} \mathrm{~F}$. Direct stimulation at a fast rhythm led to post-hatching development into normal fast type while a slow rhythm influenced the development of denervated PLD towards the slow type. In innervated PLD, the effect of stimulation at a slow rhythm was less important than in denervated PLD. These results suggest that the rhythm of the neural and/or contractile activity plays an important role in the MLC expression during embryonic and post-natal development of the chicken fast muscle.

\section{Introduction.}

De nombreux faits expérimentaux tendent à prouver que la nature des isoformes de myosine exprimées par le muscle est dépendante de l'innervation. Chez le mammifère adulte, les expériences de réinnervation croisée et de stimulation indirecte montrent que la synthèse des chaînes légères de myosine (MLC) de type lent ou rapide est dépendante de l'activité imposée par le motoneurone (Sréter et al., 1974 ; Weeds et al., 1974 ; Gauthier et al., 1983 ; Pette et al., 1976 ; Brown et al., 1983). L'influence de la nature de l'innervation sur la synthèse des MLC est aussi mise en évidence par les expériences de réinnervation croisée chez le poussin nouveau-né (Syrový et Zelená, 1975). Les mécanismes qui régulent l'expression des MLC au cours du développement embryonnaire demeurent à élucider. Aux stades précoces du développement chez l'oiseau elle paraît être indépendante de l'innervation (Crow et Stockdale, 1986 ; Phillips et al., 1986). Ensuite l'activité motrice est nécessaire à la synthèse des MLC lentes 
(Gauthier et al., 1984 ; Crow et Stockdale, 1986). Dans des travaux antérieurs nous avons montré qu'une stimulation médullaire chronique à rythme lent ralentit la contraction du PLD, modifie la topologie synaptique, la typologie des fibres musculaires et certaines activités enzymatiques (Renaud et al., 1978; Toutant et al., 1980 ; Renaud et al., 1983 ; Fournier Le Ray et al., 1986). Ceci nous a conduits à étudier les répercussions de telles stimulations médullaires, à rythme lent ou rapide, sur l'accumulation des MLC dans le muscle PLD de l'embryon.

De plus, dans le but d'appréhender, pour un muscle en cours de maturation, la contribution de l'activité contractile dans l'expression des MLC, nous avons étudié les effets d'une stimulation électrique directe, à rythme lent ou rapide, du PLD de jeunes poussins, normalement innervés ou après dénervation.

\section{Matériel et méthodes.}

Stimulation médullaire chronique chez l'embryon. - La stimulation a été faite selon la méthode de Renaud et al., (1978). Elle a été imposée du $10^{\mathrm{e}}$ au $16^{\mathrm{e}}$ jour soit à fréquence lente $(0,5 \mathrm{~Hz}$, chocs isolés de $5 \mathrm{~ms})$, soit à la fréquence rapide (10 Hz, salve d'1 min toutes les $6 \mathrm{~min}$ ).

Stimulation électrique chronique des muscles dénervés et innervés. - La stimulation a été faite selon la méthode de Khaskive et al. (1986), à raison de $12 \mathrm{~h}$ par jour et jusqu'au $14^{\mathrm{e}}$ jour après l'éclosion. Le PLD a été stimulé soit à fréquence lente $(5 \mathrm{~Hz}$, salve de $1600 \mathrm{~ms}$ toutes les $7 \mathrm{~s})$, soit à fréquence rapide $(40 \mathrm{~Hz}$, salve de $200 \mathrm{~ms}$ toutes les $7 \mathrm{~s}$ ). Les deux types de stimulation fournissent le même nombre de stimuli (environ 50000 par jour). Les muscles innervés ont été stimulés à fréquence de $5 \mathrm{~Hz}$ selon la même procédure.

Analyse électrophorétique des MLC. - Les MLC ont été séparées à partir d'extraits de protéines myofibrillaires enrichis en actomyosine (Gardahaut et al., 1985), par la technique d'électrophorèse bidimensionnelle (O'Farrell, 1975). L'analyse quantitative a été faite par densitométrie. Deux extraits myofibrillaires sont étudiés pour chaque cas examiné. Chaque extrait est préparé à partir de $200 \mathrm{mg}$ de tissu frais ( 200 muscles chez l'embryon de 12 jours, 10 à 20 muscles chez le poussin de 14 jours). Les nombres ( $n$ ) des histogrammes indiquent les nombres de gels réalisés à partir de deux extraits de protéines myofibrillaires.

\section{Résultats.}

Au $12^{\mathrm{e}}$ jour de l'embryogenèse le PLD accumule des MLC lentes $\left(\mathrm{LC}_{1} \mathrm{~S}\right.$, $\left.L C_{2} S\right)$ et rapides $\left(L C_{1} F, L C_{2} F\right)$. Les isoformes rapides prédominent $(70 \%$ du total), mais $L C_{3} F$ n'est pas présente (fig. $1 \mathrm{~A}$ ). Ultérieurement, la synthèse des $\mathrm{MLC}$ lentes est réprimée, seule $\mathrm{LC}_{2} \mathrm{~S}$ subsiste au $18^{\mathrm{e}}$ jour (fig. $1 \mathrm{C}$ ) à taux faible ( $2 \%)$. Après l'éclosion, le PLD ne contient plus que des MLC rapides (fig. 1D, E). 
$\mathrm{LC}_{3} \mathrm{~F}$ apparaît au $18^{\mathrm{e}}$ jour du développement embryonnaire et sa proportion $s^{\prime}$ accroît après l'éclosion tandis que la proportion de $\mathrm{LC}_{1} \mathrm{~F}$ diminue (fig. $1 \mathrm{E}$ ).

Effets d'une stimulation médullaire chronique chez l'embryon; - La stimulation à rythme lent $(0,5 \mathrm{~Hz})$ augmente la synthèse des $M L C(p<0,001)$ lentes dans le PLD de l'embryon (fig. 2A, B). Cette augmentation intéresse $L_{1} S$ $\left(12,4 \%\right.$ versus «traces » chez le contrôle) et $\mathrm{LC}_{2} \mathrm{~S}(13,3 \%$ versus $2,2 \%)$. La stimulation à rythme rapide $(10 \mathrm{~Hz})$ induit une synthèse précoce de $\mathrm{LC}_{3} \mathrm{~F}$ dans le PLD (fig. 2C). $L C_{3} F$, absente du muscle contrôle à 16 jours, est, après stimulation, accumulée en proportion 3 fois plus élevée que dans le PLD d'un embryon contrôle de 18 jours.

Effets d'une stimulation directe du muscle PLD dénervé et innervé de poussin. - La dénervation réprime l'accumulation de $\mathrm{LC}_{3} \mathrm{~F}(5,8 \%$ contre $19,7 \%$, $\mathrm{p}<0,001$ ) (fig. $3 \mathrm{~A}, \mathrm{~B}$ ). Tandis que les muscles PLD contrôle et dénervé sont dépourvus de MLC lentes, elles sont synthétisées dans le PLD dénervé stimulé à rythme lent (fig. $3 C$ ) et la synthèse de $L_{3} F$ est partiellement restaurée. Une activité contractile à rythme rapide imposée au PLD dénervé ne provoque pas la synthèse de MLC lentes mais modifie les proportions relatives des MLC rapides par comparaison au muscle normal ou dénervé (fig. 3A, B, D). Ainsi la synthèse de $L_{3} F$ est augmentée et celle de $L C_{1} F$ réduite $(p<0,01)$. Une stimulation à rythme lent du PLD innervé provoque une néosynthèse d'isoformes lentes (fig. $3 E$ ) qui est très inférieure (traces) à celle du PLD dénervé stimulé au même rythme (fig. $3 C$ ). L'accumulation de $L_{3} F$ est diminuée $(p<0,01$ ) (fig. $3 A, E$ ) comme dans le cas du PLD dénervé stimulé à rythme lent (fig. $3 \mathrm{C}$ ).

\section{Discussion.}

La différenciation du PLD vers le type adulte rapide consiste en la répression progressive de la synthèse des MLC lentes et en l'apparition en fin de période embryonnaire de l'isoforme rapide $\mathrm{LC}_{3} \mathrm{~F}$ dont l'accumulation augmente après l'éclosion au détriment de celle de $L_{1} C_{1} F$. Les effets de la stimulation médullaire chronique chez l'embryon montrent que l'expression des MLC est influencée par le rythme d'activité. En effet, une stimulation à rythme lent accroît la synthèse des MLC lentes dans le futur muscle rapide PLD, tandis qu'une stimulation à rythme rapide accélère la synthèse de $L_{3} F$ et ainsi la différenciation du PLD de l'embryon vers le type adulte. La stimulation médullaire chronique ne permet pas de discriminer les effets du rythme de l'activité neuronale de ceux de l'activité contractile. C'est pourquoi nous avons examiné la synthèse des MLC dans le PLD dénervé de jeune poussin stimulé à rythme lent ou rapide. Un rythme rapide permet une maturation du muscle vers le type rapide (augmentation de $\mathrm{LC}_{3} \mathrm{~F}$ et diminution de $L C_{1} F$ ). Par contre, un rythme lent oriente la maturation vers le type lent (néosynthèse des MLC lentes). Appliquée au muscle innervé la stimulation s'avère moins efficace pour induire la synthèse de MLC lentes. Ceci résulte vraisemblablement du fait qu'en présence du nerf la stimulation surimpose ses effets à ceux de l'activité spontanée qui n'est pas supprimée. 

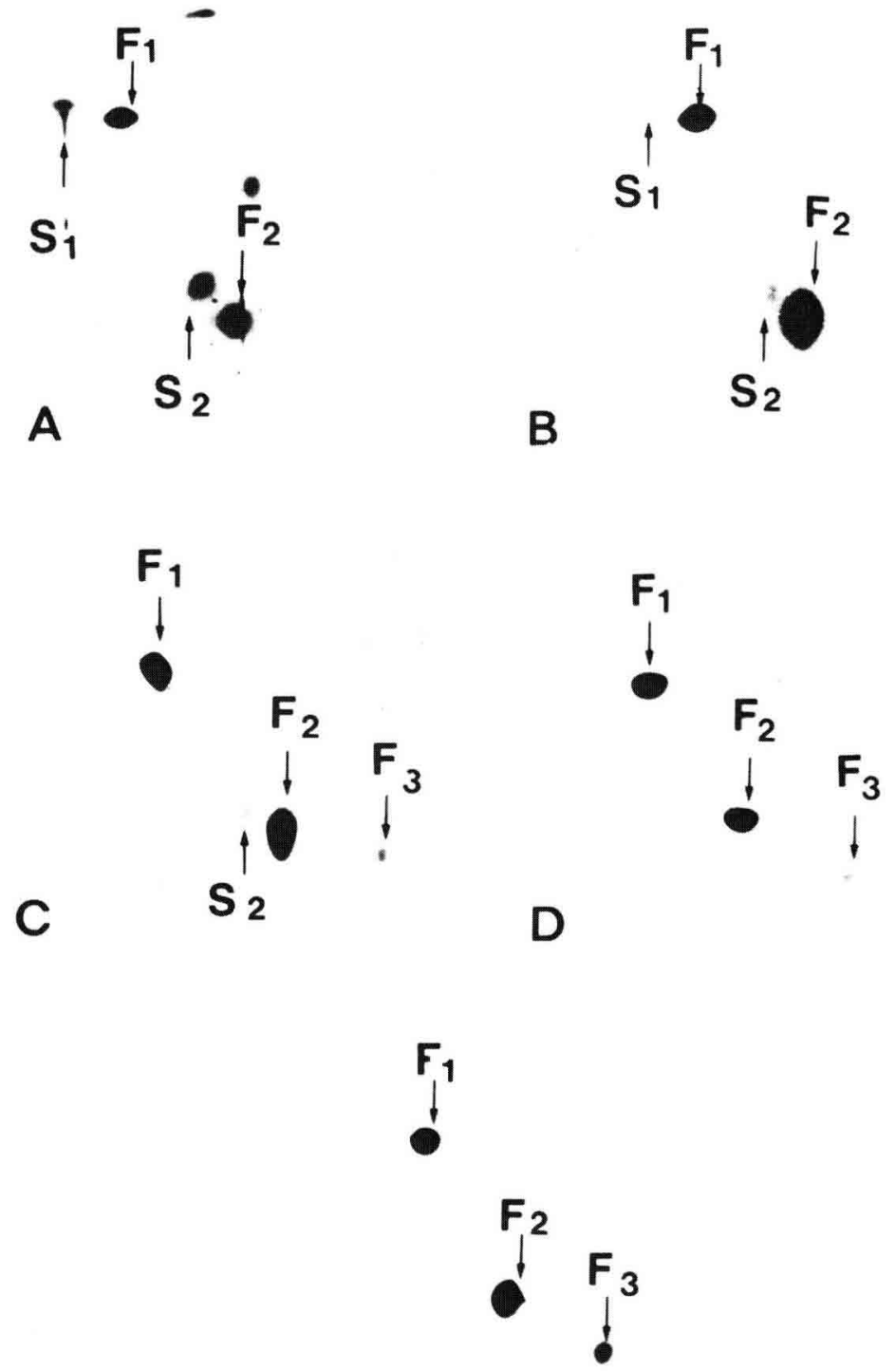

$E$

FIG. 1. - Ge/s d'électrophorèse de deuxième dimension des chaînes légères de myosine extraites du muscle PLD. (A) embryon de 12 jours; (B) embryon de 16 jours; (C) embryon de 18 jours : (D) poussin de 2 jours ; (E) poussin de 14 jours.

$S_{1}: L C_{1} S ; S_{2}: L C_{2} S ; F_{1}: L C_{1} F ; F_{2}: L C_{2} F ; F_{3}: L C_{3} F$ 

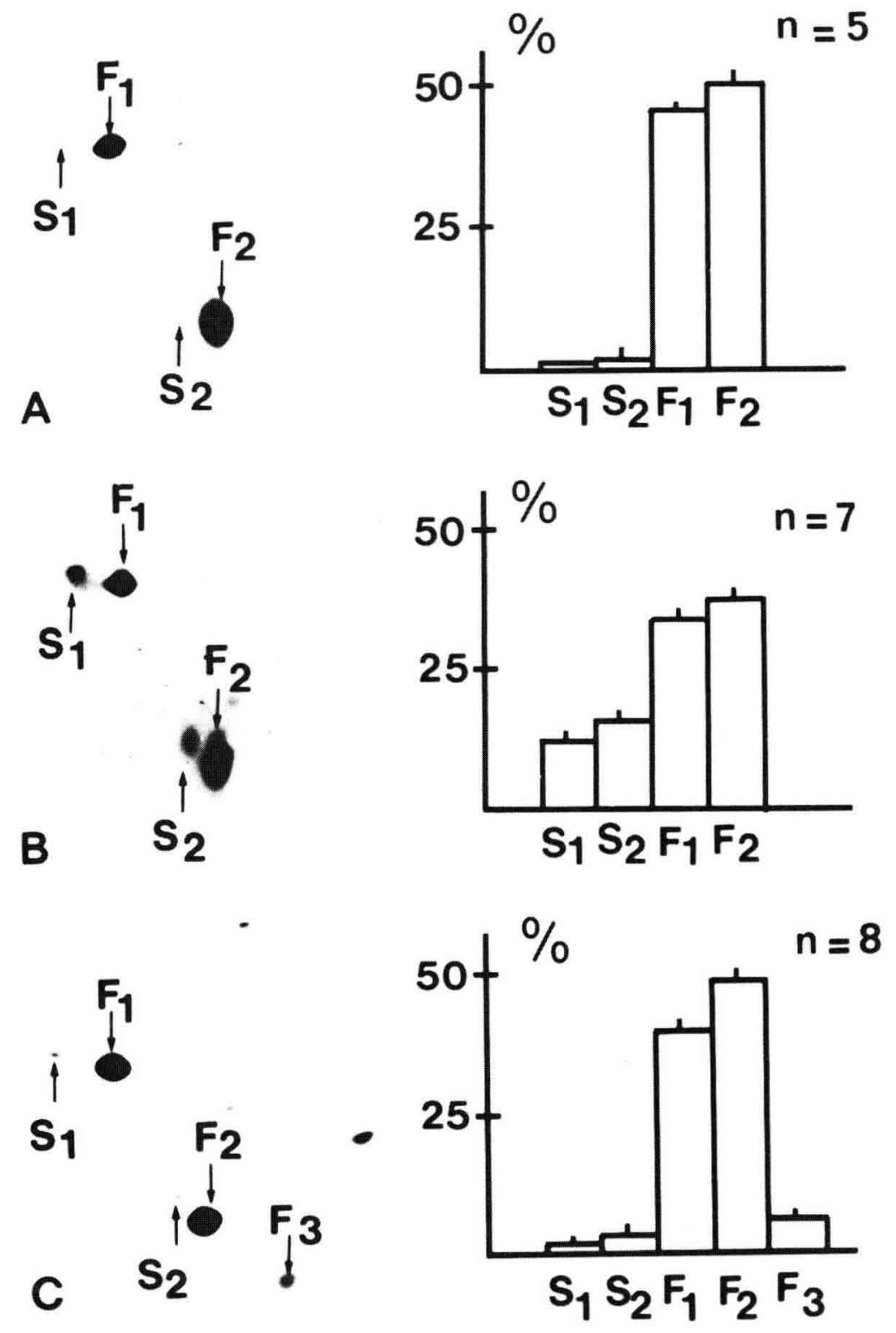

FIG. 2. - Effets de stimulations médullaires chroniques à rythme lent ou rapide sur la composition des chaînes légères de myosine du muscle PLD de l'embryon de poulet de 16 jours.

(A) embryon contrôle; (B) stimulation à rythme lent $(0,5 \mathrm{~Hz})$; (C) stimulation à rythme rapide $(10 \mathrm{~Hz})$.

A droite, histogrammes de la quantification des gels (valeurs moyennes \pm s.e.m.) 
A

$F_{1}$

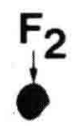

$F_{3}$

B<smiles>[3H][IH-]</smiles><smiles>F</smiles><smiles>[PH2+]</smiles><smiles>[AsH2]C=[AsH2]</smiles>

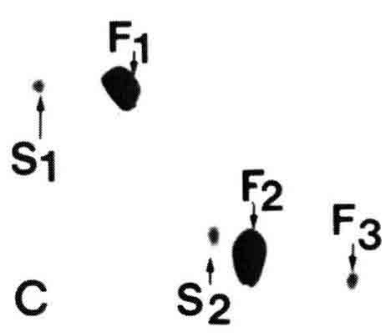<smiles></smiles>

D
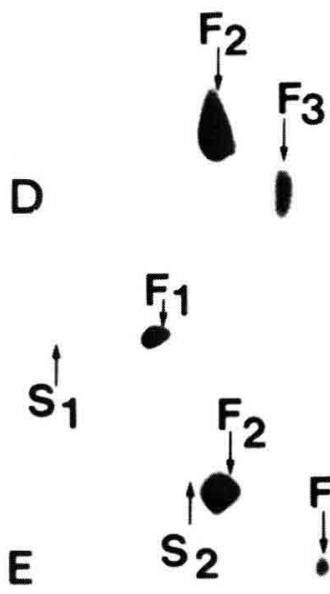

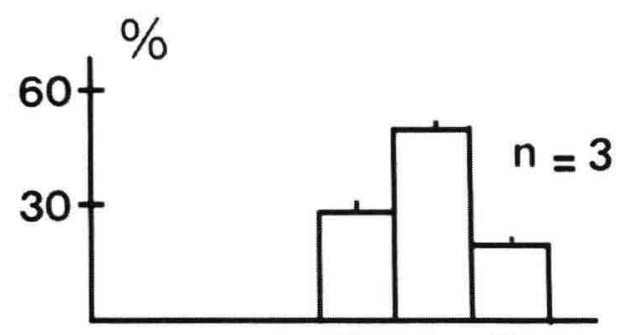

$\begin{array}{llll}F_{1} & F_{2} & F_{3}\end{array}$

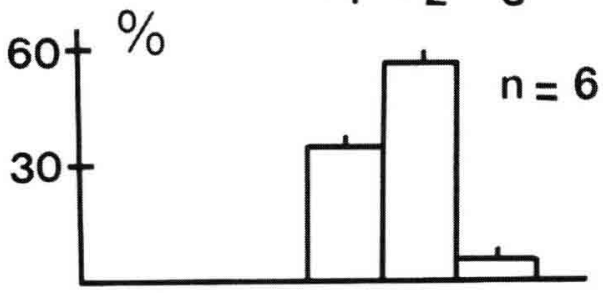

$\begin{array}{llll}F_{1} & F_{2} & F_{3}\end{array}$
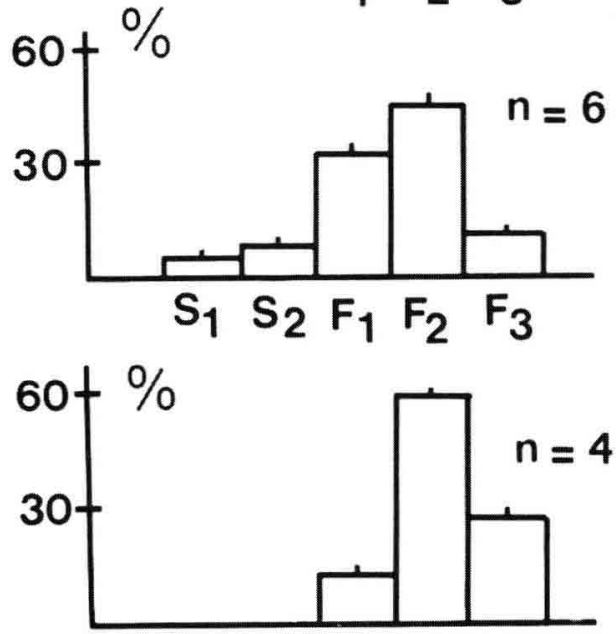

$\begin{array}{llll}F_{1} & F_{2} & F_{3}\end{array}$

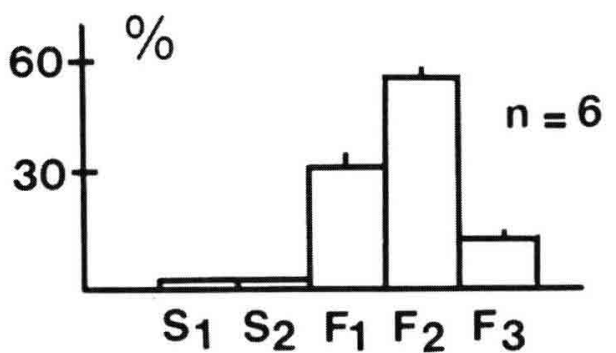

FIG. 3. - Effets surl'expression des chaînes légères de myosine de stimulations directes à rythme lent ou rapide appliquées aux muscles $P L D$ de poussin. Début de la stimulation au $2^{\mathrm{e}}$ jour, prélèvement des muscles à 14 jours.

(A) PLD contrôle ; (B) PLD dénervé ; (C) PLD dénervé-stimulé $5 \mathrm{~Hz}$; (D) PLD dénervé-stimulé $40 \mathrm{~Hz}$; (E) PLD innervé-stimulé $5 \mathrm{~Hz}$;

A droite, histogrammes de la quantification des gels (valeurs moyennes \pm s.e.m.). 
Ces résultats montrent que la synthèse des MLC dans le développement embryonnaire et post-natal du muscle rapide d'oiseau est dépendante du rythme de l'activité nerveuse et/ou contractile.

Un récent travail de Moss et al. (1986) souligne l'existence d'une corrélation entre l'activité contractile et le contenu en $\mathrm{LC}_{3} \mathrm{~F}$ de fibres musculaires en culture. Chez l'embryon la stimulation centrale à rythme rapide induit une synthèse de $\mathrm{LC}_{3} \mathrm{~F}$ et chez le poussin le taux de $\mathrm{LC}_{3} \mathrm{~F}$ du muscle dénervé stimulé est plus élevé pour un rythme rapide que pour un lent. Ce taux baisse même par stimulation à rythme lent du PLD dénervé. Au total, il apparaît que le taux de $\mathrm{LC}_{3} \mathrm{~F}$ est influencé par le niveau d'activité et par son rythme. Le fait que le muscle PLD, chez l'embryon comme chez le jeune poulet, accumule $\mathrm{LC}_{3} \mathrm{~F}$ en proportion d'autant plus importante qu'il manifeste une activité contractile de type rapide suggère l'existence d'une relation possible entre la vitesse de contraction des fibres musculaires et leur contenu en $\mathrm{LC}_{3} \mathrm{~F}$.

$13^{e}$ Réunion du groupe Développement I.N.R.A., Cap d'Agde, 25-27 mai 1987.

Remerciements. - Ce travail a été rendu possible par une aide de l'Association des Myopathes de France.

\section{Références}

BROWN W. E., SALMONS S., WHALEN R. G., 1983. The sequential replacement of myosin subunit isoforms during muscle type transformation induced by long terme electrical stimulation. $J$. biol. Chem., 258, 14686-14692.

CROW M. T., STOCKDALE F. E., 1986. Myosin expression and specialization among the earliest muscle fibers of the developing avian limb. Dev. Biol., 113, 238-254.

FOURNIER LE RAY C., RENAUD D., LE DOUARIN G. H., 1986. Effect of spinal cord stimulation on the metabolism of developing latissimus dorsii muscles in chick embryo. Cell Diff., 19. 225-228.

GARDAHAUT M. F., ROUAUD T., RENAUD D., LE DOUARIN G. H., 1985. Influence of spinal cord stimulation upon myosin light chain and tropomyosin subunit expression in fast muscle (posterior latissimus dorsi) of the chick embryo. J. Musc. Res. Cell Motility, 6, 769-781.

GAUTHIER G. F., BURKE R. E., LOWEY S., HOBBS A. W., 1983. Myosin isozymes in normal and cross-reinnervated cat skeletal muscle fibers. J. Cell Biol., 97, 756-771.

GAUTHIER G. F., ONO R. D., HOBBS A. W., 1984. Curare-induced transformation of myosin pattern in developing skeletal muscle fibers. Dev. Biol., 105, 144-154.

KHASKIYE A., RENAUD D., LE DOUARIN G. H., 1986. Effects of denervation and direct electrical stimulation upon the post-hatching differentiation of posterior latissimus dorsi muscle in chicken. Cell Diff., 18, 27-35.

MOSS P., MICOU-EASTWOOD J., STROHMAN R., 1986. Altered synthesis of myosin light chains is associated with contractility in cultures of differentiating chick embryo breast muscle. Dev. Biol., 114, $311-314$.

O'FARRELL P. H., 1975. High resolution two-dimensional electrophoresis of proteins. J. biol. Chem., 250, 4007-4021.

PETTE D., MÜLLER W., LEISNER E., VRBOVÁ G., 1976. Time dependent effects on contractile properties, fiber population, myosin light chains and enzymes of energy metabolism in intermittent and continuously stimulated fast twitch muscle of the rabbit. Pflügers Arch., 364, 103-112. 
PHILLIPPS W. D., EVERETT A. W., BENNETT M. R., 1986. The role of innervation in the establishment of the topographical distribution of primary myotube types during development. J. Neurocytol., 15, 397-405.

RENAUD D., LE DOUARIN G. H., KHASKIYE A., 1978. Spinal cord stimulation in chick embryo: effects on development of the posterior latissimus dorsi muscle and neuromuscular junctions. Exp. Neurol., 60, 189-200.

RENAUD D., GARDAHAUT M. F., ROUAUD T., LE DOUARIN G. H., 1983. Influence of chronic spinal cord stimulation upon differentiation of $\beta$ muscle fibers in a fast muscle (posterior latissimus dorsi) of the chick embryo. Exp. Neurol., 80, 157-166.

SRÉTER F. A., GERGELY J., LUFF A. L., 1974. The effect of cross-reinnervation on the synthesis of myosin light chains characteristic of slow muscle in response to long-term stimulation. Biochem. biophys. Res. Commun., 56, 84-89.

SYROVY I., ZELENA J., 1975. The onset and progress of transformation of avian slow into fast muscles under neural influence. Pflügers Arch., 360, 121-134.

TOUTANT M., BOURGEOIS J. P., TOUTANT J. P., RENAUD D., LE DOUARIN G. H., CHANGEUX J. P., 1980. Chronic stimulation of the spinal cord in developing chick embryo causes the differentiation of multiple clusters of acetylcholine receptor in the posterior latissimus dorsii muscle. Dev. Biol., 76, 384-395.

WEEDS A. G., TRENTHAM D. R., KEAN C. J. C., BULLER A. J., 1974. Myosin from crossreinnervated cat muscles. Nature, 247, 135-139. 